\title{
Innovative Electron Microscopy for Multi-Layer van der Waals Heterostructures Quantum Materials Discovery
}

David Bell ${ }^{1}$, Cigdem Ozsoy-Keskinbora ${ }^{2}$, Austin Akey ${ }^{1}$, Aravind Devarakonda ${ }^{3}$ and Joseph
Checkelskey $^{3}$

${ }^{1}$ Harvard University, Cambridge, Massachusetts, United States, ${ }^{2}$ Thermo Fisher Scientific, Achtseweg Noord 5, 5651 GG Eindhoven, The Netherlands, Eindhoven, Noord-Brabant, Netherlands, ${ }^{3}$ MIT, United States

The discovery of extraordinary new quantum materials with striking properties has caused great excitement and promises to transform signal processing and computation. We have performed integrated research on three materials. Remarkably, the quantum phenomena displayed by these materials persists at room temperature, changing the rules for signal processing and computation and opening the way for quantum electronics.

It is possible to form via controlled growth systems heterointerfaces in bulk materials, we present the example of our newly synthesized material $\mathrm{Ba}_{6} \mathrm{Nb}_{11} \mathrm{~S}_{28}$ (see Fig. 1a) [1]. This material naturally realizes vdW coupled heterointerfaces between transition metal dichalcogenide (TMD) monolayers (hexagonal $\mathrm{NbS}_{2}, H-\mathrm{NbS}_{2}$ ) and insulating spacers $\mathrm{Ba}_{3} \mathrm{NbS}_{5}$ (see Fig. 1b). TEM diffraction taken along the c-axis shows that the hexagonal spacer and TMD layers, (Fig. 1c\&d), orange and blue, respectively) are commensurate. The electronic band structure can be understood as that resulting from superimposing a periodic potential defined by $\mathrm{Ba}_{3} \mathrm{NbS}_{5}$ onto monolayer $H-\mathrm{NbS}_{2}$. This is similar to the mechanism which yields flatbands and strongly correlated physics in twisted-bilayer graphene and TMD heterostructures. Low Voltage Aberration-corrected electron microscopy has been used to characterize grown materials with high resolution at low beam voltages $(40 \& 80 \mathrm{kV})$ to directly visualize structural defects and relate them to performance [2].

We have also examined Kagome type 2-D materials Using angle-resolved photoemission, we have also detected a pair of correlated Dirac cones near the Fermi level with a $30 \mathrm{meV}$ mass gap acting as a source of Berry curvature in a $\mathrm{Fe}_{3} \mathrm{Sn}_{2}$ kagome bilayer structure [3\&4]. We show this behavior is a consequence of the underlying symmetry properties of the bilayer kagome lattice in the spin-orbit coupled ferromagnetic state. This offers insight into recent discoveries of exotic electronic behavior in kagome lattice antiferromagnets and provides a steppingstone toward lattice model realizations of fractional topological quantum states in other materials systems.

The imaging and analysis of quantum materials presents new challenges on how to minimize surface and sample damage while imaging and analyzing structures at the direct atomic level, new approaches are needed in order to corelate materials properties with structure, we present some of our multi-modal and multi techniques approach in this presentation.

This work was supported by the STC Center for Integrated Quantum Materials, NSF Grant No. DMR1231319. Portions of this work was performed at the Center for Nanoscale Systems (CNS), a member of the National Nanotechnology Coordinated Infrastructure Network (NNCI), which is supported by the National Science Foundation under NSF award no. 1541959. 
a

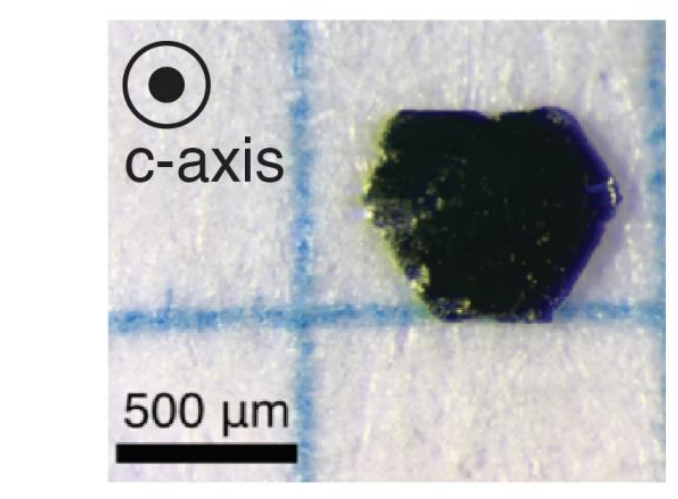

C

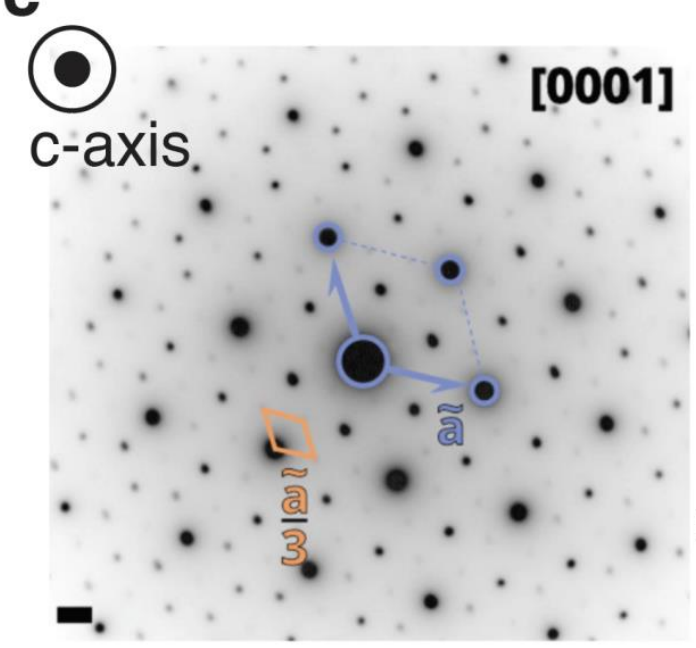

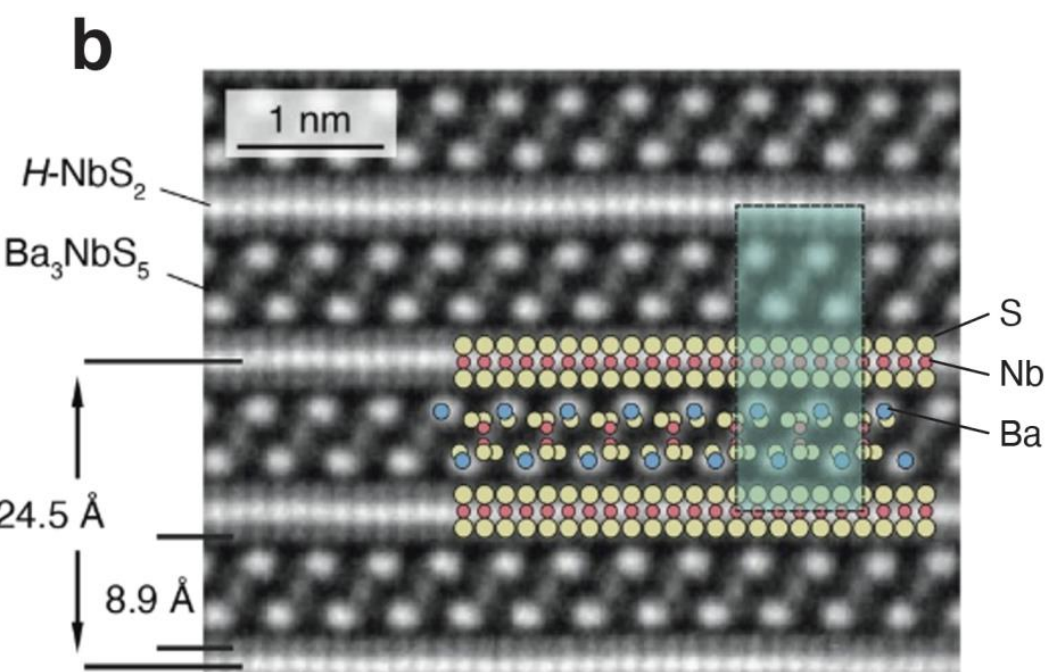

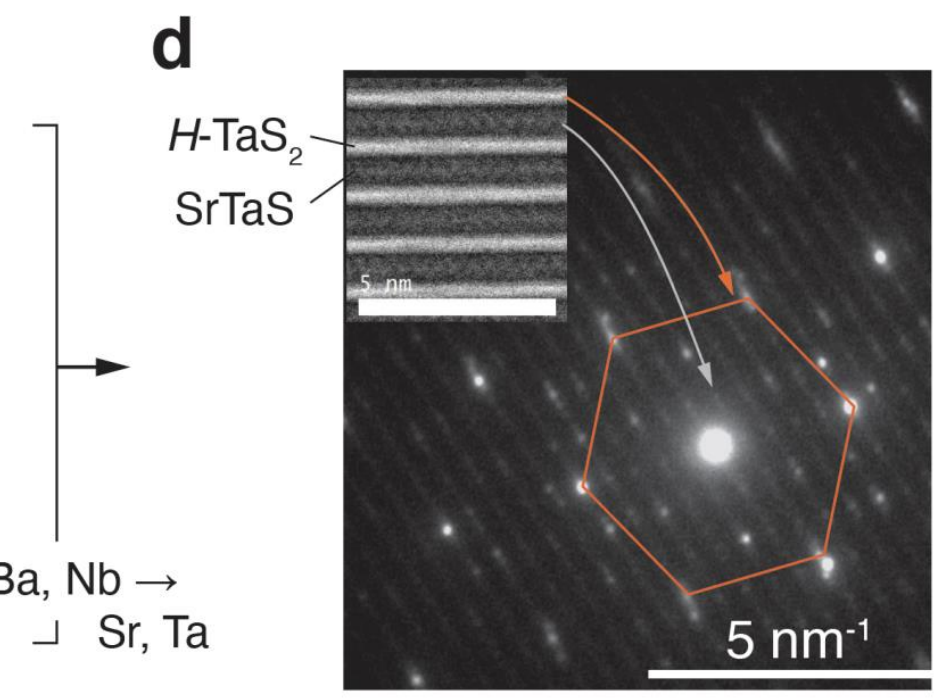

Figure 1. Figure 1 - (a) Optical image of Ba6Nb11S28 crystal (b) High-resolution TEM cross-section showing alternating $\mathrm{H}-\mathrm{NbS} 2$ and Ba3NbS5 layers. (c) TEM diffraction image of Ba6Nb11S28 sighted along the c-axis. (d) inset, High-resolution TEM cross-section of H-TaS2 containing compound. main, TEM diffraction sighted along c-axis of $\mathrm{H}-\mathrm{TaS} 2$.

\section{References}

[1] Devarakonda A, Inoue H, Fang S, Ozsoy-Keskinbora C, Suzuki T, Kriener M, Fu L, Kaxiras E, Bell DC, Checkelsky JG.. Science Oct 9;370(6513):231-236 (2020).

[2] Bell D, Erdman N. Low Voltage Electron Microscopy. Bell D, Erdman N, editors. Chichester, UK: John Wiley \& Sons, Ltd; (2012)

[3] Kang M, Ye L, Fang S, You JS, Levitan A, Han M, Facio JI, Jozwiak C, Bostwick A, Rotenberg E, Chan MK, McDonald RD, Graf D, Kaznatcheev K, Vescovo E, Bell DC, Kaxiras E, van den BrinkJ, Richter M, Prasad Ghimire M, Checkelsky JG, Comin R. Nature Materials 2020 Feb;19(2):163-169.

[4] L. Ye, M. Kang, J. Liu, F. von Cube, C. R. Wicker, T. Suzuki, C Jozwiak, A. Bostwick, E. Rotenberg, D. C. Bell, L. Fu, R. Comin, and J. G. Checkelsky, Massive Dirac fermions in a ferromagnetic kagome metal, Nature 555, 638-642 March (2018). 